Advanced Computing: An International Journal ( ACIJ ), Vol.3, No.1, January 2012

\title{
MULTI-AGENTS SIMULATION OF HUMAN BEHAVIOR IN A SITUATION OF EMERGENCY
}

\author{
Benkhedda Samira $^{1}$ and Bendella Fatima ${ }^{2}$ \\ SIMPA Laboratory, Dept of computer science, Faculty of Science, University of \\ Science and Technology of Oran-USTO, Algeria. BP 1505, 31000 \\ ${ }^{1}$ benkhedda. usto@gmail.com, ${ }^{2}$ bendella@univ-usto.dz
}

\begin{abstract}
The simulation has long been dominated by approaches based on the equations, which do not describe the behavior of the system, until the emergence of approaches oriented agents. These multi-agent systems are increasingly used to study the complexity of the natural or social phenomena. In this context, the simulations are based on agent-oriented representation to describe the characteristics of the situation that all actions can be performed by actors. These tools have been used in situations of crises that are usually very difficult to manage, because of their complexity or the damage on help. In this paper, we propose a new approach for simulating multiple agents in a medical emergency based on practical reasoning of human and using the notion of simulation of complex systems.
\end{abstract}

\section{KEYWORDS}

Multi agents systems, Medical emergency, Multi agent simulation.

\section{INTRODUCTION}

Multi-agent systems are used to study the complex natural and social phenomena.

In this paper we present our simulation of a medical emergency that is 'heart attack', because if no action is taken immediately in emergency assistance and if do not act soon, the victim's life is in danger in a short term. In this context, we propose a multi-agent simulation and MLearning application that is flexible and intuitive enough to allow the succourer to save the life of a victim of heart attack because the speed processing is very important in this case. So, we propose a new approach for multi-agent systems in a medical emergency based on practical reasoning of human beings and using the notion of the simulation of complex systems, so we set up a multi agents simulation and during this simulation, the active actor of the system will learn through a mobile application and it will enrich its knowledge base of a cognitively adapting to changes in its environment and changes interactions between agents of the model.

\section{CONCEPT OF MULTI AGENTS SIMULATION}

The multi-agent simulation is a simulation that employs the concept of multi-agents systems in the conceptualization, specification and implementation. A multi-agents system simulated living in a simulated environment; the multi-agents simulation directly represents the people, their behavior, their actions in the environment and their interactions. The multi-agents simulation is Interactions, Agents and Environment [1].

Ferber [2] notes that the multi-agents simulation allows the study of complex systems. It represents the complexity of a phenomenon through the interaction of a single set of entities called agents. Each agent can:

1) Communicate with other agents to exchange information.

DOI : $10.5121 /$ acij.2012.3102 
2) Perceive and act on all or part of the environment.

3) Apply knowledge, skills and other resources to perform their individual personal goals.

The objective of the multi-agents simulation is:

1) to infer the nature of the functioning of the entities of a complex system.

2) System Analysis.

Simulator knows two phases of use:

1) A research phase in which the simulator acts as an incubator model.

2) An operational phase: once the model is validated, the simulator becomes a tool in the field.

\section{SiMULATION OF COMPLEX SYSTEM}

Simulate a complex system is to model its components, their behaviors and interactions between them and with their environment and then run the model obtained numerically.

A feature of these systems is that one cannot predict the evolution of the modelled system without going through this phase of simulation. The approach "experimental" simulation makes it possible to reproduce and to observe complex phenomena (biological or social) in order to understand and anticipate their evolution [3].

\section{MULTI-AGENTS SIMULATION IN THE CASE OF HEART ATTACK}

\subsection{Heart attack [4]}

A heart attack is a serious problem caused by a blood clot in a coronary artery or one of the chambers of the heart. Cardiac arrest is the sudden stoppage of the heart that pumps more blood.

\subsection{Signs of heart attack [4]}

The cardiopulmonary resuscitation (CPR) quickly can save lives. Prompt treatment to break up clots can greatly increase the chances of survival of the person who suffers of heart attack. Since prompt treatment can make a difference, it is important to know the early signs of heart attack.

In case of heart attack, you may experience one or more of the following:

1) Discomfort in the center of the chest that lasts more than 5 minutes or comes and goes. It takes the form of an uncomfortable pressure, tightness, a feeling of heaviness or pain.

2) Discomfort in other parts of the body, such as pain or discomfort in one or both arms, neck, at the jaw or stomach.

3) Shortness of breath is often accompanied by chest discomfort but can occur before the discomfort. Among other signs, call the cold sweats, nausea and a sensation of floating.

The CPR is a combination of chest compressions with rescue breathing done on the victims is believed to be in cardiac arrest. When cardiac arrest occurs, the heart stops pumping blood. CPR can support a small amount of blood flow to the heart and brain to "buy time" to restore normal heart function [5]. 


\section{MOBILE-LEARNING [6]}

Mobile-learning is a logical extension of e-learning. In this sense it refers to the provision of courses or learning objects through mobile devices such as Pocket PC, cell phones or the Palm Pilot Users will have lessons in reduced format, but the main advantage of such a solution is accessible at any time of day and from any location with a network is nearby.

Today the knowledge of students in mobile technology (accessibility, ease of use, speed of adaptation) makes the m-learning possible.

\section{COMPLEX SIMUL}

The main objective of our project (Complex SIMUL) is to simulate an emergency based on the simulation of complex systems and using the reasoning of human, which is the The major disadvantage in the ancient works of emergency like IDESS (Infectious Disease Epidemic Simulation System) [7], the cognitive of agents is ignored.

Complex SIMUL includes reactive agents that will work in a complementary way and deliberative agents (BDI type) that will incorporate the concept of practical reasoning in humans. Our system includes three different types of agents (figure1):

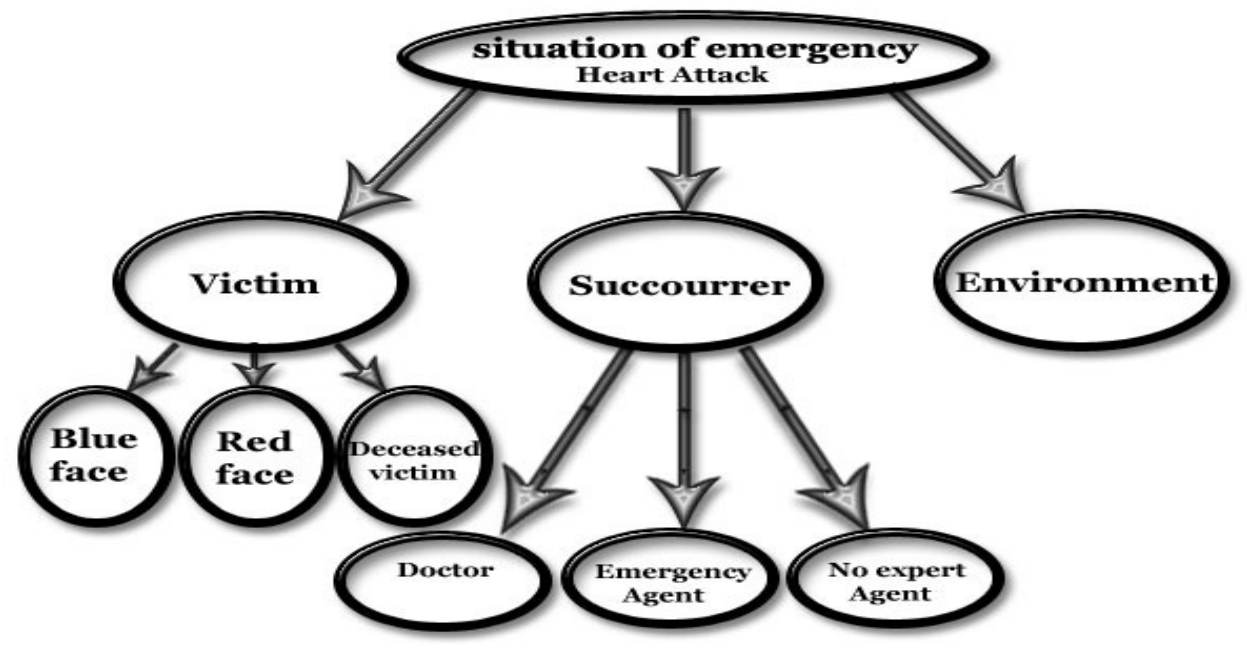

Figure1: Architecture of Complex SIMUL.

\subsection{Victim Agent}

It is a passive actor, it can take three different cases (a patient with a blue face, red or a deceased victim), it is reactive, and it reacts to the actions of the agent rescuer (move, help him ...).

\subsection{Environment Agent}

It is the agent that identifies the location of the accident, it is reactive type and it is dynamic (the road, the greenery ...).

\subsection{Succourer Agent}

It is the active player of our emergency; there are three types of agents:

1) The emergency agent and the doctor agent: aid workers are expert-like agent, they are reactive agents.

2) The no-expert agent: an agent's type BDI (Belief, Desire and intention), the agent has beliefs about the world in which it operates, and it must meet the desires by making intentions). 


\section{WHY A BDI AGENT TYPE?}

This architecture is the most valued. In theoretical point of view, a BDI agent can perform any type of task; the architecture allows the agents to solve complex problems.

In this architecture, agents have a feature that allows evaluating the utility of each action. Contrary to the cognitive architecture of agents, the agents ask random actions when they were not able to achieve their goals. BDI agents can determine the action (or actions) to be performed to get as close as possible goal. This means that when an agent can achieve its goal by setting an action, then it will select the action as close as possible to the goal or action which will achieve this goal as quickly and it must also be some kind of rational process for deciding what intentions select as appropriate [8].

\section{THE OPERATION OF OUR SUCCOURER AGENT LIKE A BDI AGENT}

Our agent includes an event queue (the actions of the agent to whom he rescued victim) by storing the internal events of the system, the beliefs (knowledge of the agent), a library of plans (know-how of the agent ), a battery of desires (goals of the agent) and a stack of intentions (instantiated plans to achieve goals) (figure2). The BDI interpreter cycle begins by updating the event queue and beliefs of the agent. It then activates new desires by selecting the plans of the library, so our agent has the opportunity to decide by running the first selected action stack of intentions, and so on.

This agent must:

1) Observe the environment agent and the victim agent for the possible risks.

2) Report a warning (phone call).

3) Select a plan (and it depends on his desires).

4) Select a plan by running the corresponding plan of action (the $\mathrm{ABC}$ of first aid).

Our no-expert agent is going to learn during the simulation using his mobile phone through the M-Learning. During the M-Learning, our BDI agent will learn along the ABC of first aid.

We used the concept of priority for emergency agents, the doctor agent has the highest priority then the emergency agent but no-expert agent will take the lowest value of priority zero boots (0) then after every successful in emergency and in the same case this value will increment to 1 and so on.

The priority of the no-expert agent is less than or equal to the priority of the emergency agent but the last two priors are always less than that of the doctor agent.

The BDI agent will protect the victim agent and his self from danger (the middle of a road) then it will use the $\mathrm{ABC}$ of first aid. 


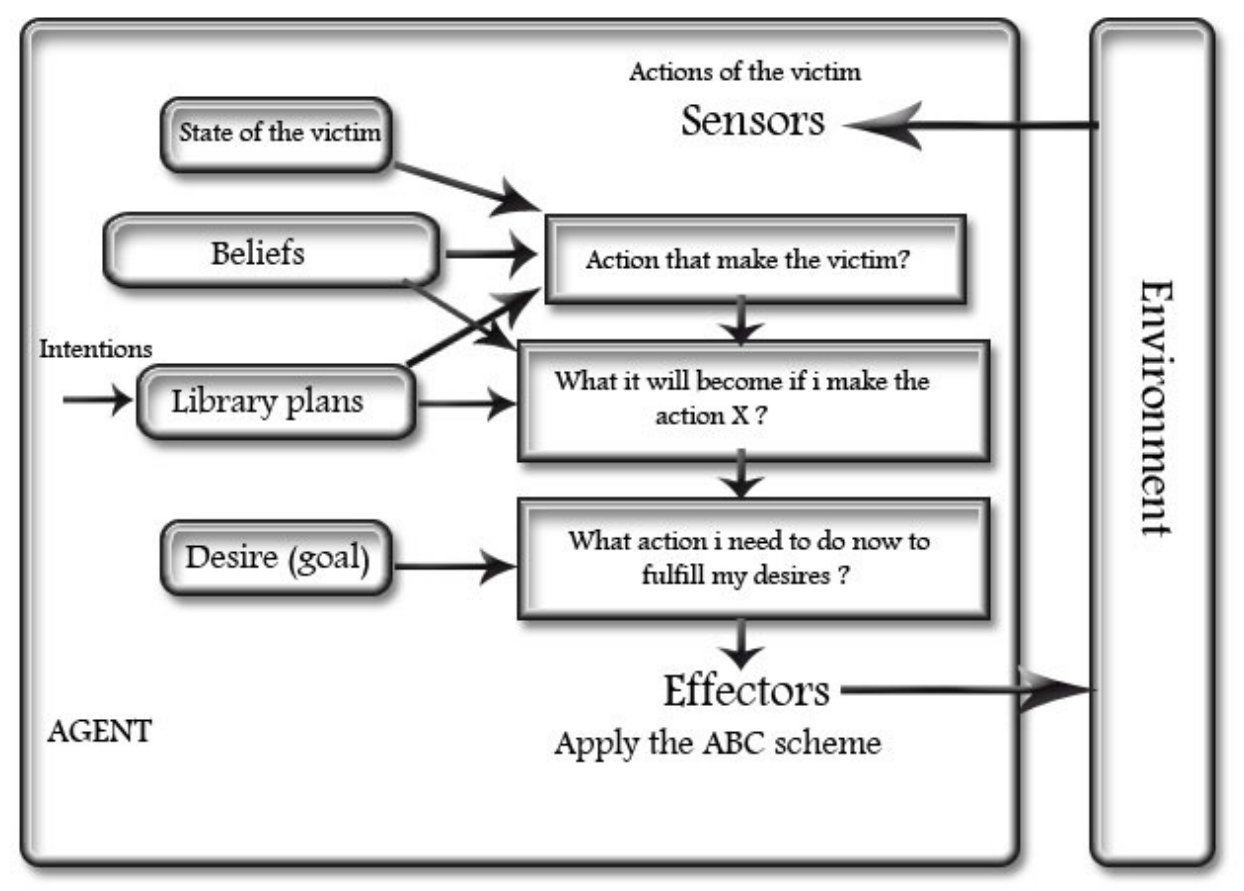

Figure2: The BDI agent of Complex SIMUL.

\section{THE MOBILE APPLICATION}

The mobile application is an interface between the user and the system, it was built in such way that it is as simple as possible, to avoid discourage the use of this application, and because as the mobile phone does not give a very wide selection in the display of data. The application is a kind of questionnaire (yes / no) that the user must meet in order to identify the step reach and receive instructions to do it in the short time, the questions may appear very simplistic, because it was assumed that the user of this application is probably a person who does not know very established in the field of aid, and so we should not use a lot of technical terms. The questionnaire was composed in three steps according to the diagram $\mathrm{ABC}$ [9]. The many participants in the course of first aid have memorized the mnemonic RRSS (he answers, he breathes, he is bleeding, his pulse is it noticeable). RRSS was purely an invention Helvetic. In the new course is the international abbreviation "ABC" (that is taught. The big difference between the old and the new scheme is that only "RRSS" we did an appreciation of the patient, while "ABC" brought immediate action, resulting in more timely assistance [10]. The ABC of first aid is the acronym of a mnemonic device for memorizing the order of first-aid treatment in case of accident [5].

A (for airways $)=$ clear the airway.

$\mathrm{B}$ (for breathing) $=$ to restart breathing.

$\mathrm{C}($ for cardiac $)=$ measuring the presence of heart rate

\section{Conclusion}

In this paper, We have tried to show the methodology for the design of our simulation, we have implemented a multi-agent simulation and an application of M-Learning which is flexible and intuitive enough to allow the rescuer to the first aid to save the life of a victim of a heart attack because the speed of treatment is very important in this case. 
Advanced Computing: An International Journal ( ACIJ ), Vol.3, No.1, January 2012

We currently implement the agents of our simulation and integrate them into a learning application.

\section{REFERENCES}

[1] Wafa Ketata, Wided Lejouad and Chaari. 2007. UneOntologie pour la réutilisation des Interactions dans un Système Multi-Agents. JFO, 18-20 octobre 2007, Sousse, Tunisie.

[2] J. Ferber, 1999. Multi-agent systems. Reading MA : Addison-Wesley.

[3] Alain Boucher, NGUYEN Nhu Van, 2007. L'interaction dans simulation multi agents. Hanoi.

[4] McKesson Provider Technologies, 2006. La crise cardiaque : Signes annonciateurs précoces.

[5] http://tilz.tearfund.org/Publications/Footsteps+1120/Footsteps+18/The+ABC+of+first+aid.htm.

[6] J, MUESSER., 2005. Conception et réalisation d'un objet pédagogique pour périphérique mobile.

[7] Dean Yergens, Julie Hiner, Jörg Denzinger ET Tom Noseworthy.2006. Multi Agent Simulation System for Rapidly Developing Infectious Disease Models in Developing Countries. JAPAN.

[8] Rao A. S., Georgeff M. P.1991. Modeling Rational Agents within a BDI-Architecture.

[9] http://www.creapharma.ch/urgence_numero.htm.

[10] Werner Bühler Bilten. 08-2003. nouveau schéma d'action : de RRSS à ABC. Article : Journal des sapeurs-pompiers suisses.

Authors

Samira Benkhedda, PHD student in SIMPA Laboratory of the computer science dept, University of Science and Technology of Oran-USTO, Algeria.

Dr. Fatima Bendella, Senior Lecturer in the Department of Computer science in USTO, she got an engineer diploma in computer science at the University of Oran in 1988, a magister of USTO in 1995 and a doctorate in 2005. She directs several theses of magister and PHD in the application of multi-agent systems in software development.

She is responsible of many research projects and a national research project. 